\title{
CrimRxiv
}

\section{Review 2 of ''I know a guy': Examining homeless income generation and spatial mobility"}

\author{
Martin Šimon
}

Published on: Jan 12, 2022

License: Creative Commons Attribution 4.0 International License (CC-BY 4.0). 
Vote: Publish pending minor changes

[For votes to count, referees must reasonably explain why they voted as they did. Thus, please explain your vote. If you voted to publish pending minor changes, specify each change, why it is needed, and, possibly, how it should/could be done.]

This is solid qualitative paper, which address important topic of homeless mobility strategies related to income. This topic is often ignored in surveys of homeless populations and almost absent in studies of human mobility in general. Exploration of spatial mobility and mobility strategies of homeless people is a powerful tool that document inhumane practices of homeless management and harm it makes every day to vulnerable population. The study, as the authors themselves acknowledge, is consistent with a previous findings, but provide a few novel updates to the existing knowledge in the field. The study could benefit from: 1) more critical evaluation of mobility measurement and 2) from more careful contextualization of findings - as the homeless mobility is highly dependent on homeless conditions in a particular place and functional structuring of urban space.

[Please put additional info below, as/if you see fit.]

Here I provide a list of minor points, which the authors could address in their paper.

1) The title should include the name of city where the study was conducted. The location is important for both mobility and income opportunities for homeless, as these are highly dependent on a particular context - urban, national.

2) The abstract could highlight the most important findings of the paper.

3) The typology of housing of homeless could be described more efficiently as it basically follows ETHOS typology by FEANTSA, which is used commonly in European context.

4) Travel distance derived from narrated places is not the best measure of spatial mobility. People can move a lot within small area or make just one trip to a more distant location. Distance traveled is not the same as a path traveled, especially if you rely on walking - and these two are not necessarily correlated. Daily activity spaces or other spatial metrics might be more useful. Also, spatial mobility depend on city size, age and gender as mobility studies have documented. 
5) The introduction to the paper is dominated by a "lists of things related", which could be written in a more readable form.

6) The sentence "A challenge for the homeless ..." imply just one-sided perspective. I miss the literature seeing homeless through citizenship perspective here.

7) RG1 - The review of survival strategies is dominated by listing criminal activities, while the non-criminal activities are not much accentuated. This is not balanced view, as the results of the study also point out.

8) RQ2 - I think that the "range of travel" indicator should be critically reflected. Ability to travel is about mobility options in urban space as well as individual capabilities and institutional opportunities.

9) RQ3 - This question is more complex as the answer depends on how movement patterns are measured. The participatory GPS tracking with the homeless we did in the past indicated, that age or types of housing shape daily spatial mobility, but the relations between variables were not simple and linear.

10) In the study design, authors should be aware, that location of homeless in a particular urban space is shaping the extent of mobility due to accessibility/availability/affordability/acceptability of mobility opportunities in space.

11) Unsheltered homeless persons (page 5) do have different mobility patterns, they even have different temporal rhythms during the day.

12) The prevalence of drug use in the sample is striking, many European studies/surveys show much lower value of substance use.

13) I very much like the thick description of Less and More mobile as it highlights often less visible barriers in daily life of homeless people.

14) The interpretation of mobility of street homeless and everyone else (page 12) could be more nuanced, as there is a clear trade off between investing resources into mobility or into place to sleep.

15) Contrasts to previous studies, e.g. Jocoy, Del Casino, could be highlighted in the introductory parts of the paper.

16) The information about income of homeless is striking and alarming, even such incomes are not sufficient to get away from homelessness. 
17) The conclusion and implications are well elaborated. I am not sure how are these novel for service providers in Vancouver, I suspect that these are know, but deliberately ignored as a part of poverty management. So the reflection of current praxis might be more critical.

18) The framing of the paper might benefit from following publications: review on homelessness by B.A. Lee in the Annals of AAPSS (693, January 2021); recent paper by Kaufman D.F. in Canadian Geographer; paper by H. Aasi and Y. Lee: Spatial analytics a missing key to ending homelessness, which provide a good list of studies on the topic; symposia papers on Mobilities and Mobilisation of the Urban Poor by R. Jaffe, C. Klaufus, F. Colombijn in IJUUR 2012; report about GPS tracking of homeless in Odensee (DK) with a follow up research using participatory GPS tracking of homeless men and women in Prague and Pilsen (CZE); and study by L. Smith, L. Foley, J. Panter on Activity Space Measurement, published in 2019 in Health and Place journal. 